\title{
OPTIMIZATION OF ELASTICALLY TAILORED TOW-PLACED PLATES WITH HOLES
}

\author{
Dawn C. Jegley ${ }^{*}$ \\ NASA Langley Research Center, Hampton, Virginia 23681 \\ Brian F. Tatting ${ }^{+}$ \\ ADOPTECH, Inc., Blacksburg, Virginia 24060 \\ Zafer Gürdal \\ Virginia Tech, Blacksburg, Virginia 24061
}

\begin{abstract}
Elastic stiffness tailoring of laminated composite panels by allowing the fibers to curve within the plane of the laminate is a design concept that has been demonstrated to be both beneficial and practical. The objective of the present paper is to demonstrate the effectiveness of stiffness tailoring through the use of curvilinear fibers to reduce stress concentrations around the hole and improve the load carrying capability of panels. Preliminary panel designs that are to be manufactured and tested were determined through design studies for flat plates without holes under axial compression using an optimization program. These candidate designs were then analyzed with finite element models that accurately reflect the test conditions and geometries in order to decide upon the final designs for manufacture and testing. An advanced tow-placement machine is used to manufacture the test panels with varying fiber orientation angles. A total of six large panels measuring three feet by six feet, each of which is used to produce four specimens with or without holes, are fabricated. The panels were machined into specimens with holes and tested at NASA Langley Research Center. Buckling response and failure of panels without holes and with two different hole dimensions are presented. Buckling and failure loads of tow-steered specimens are significantly greater than the buckling and failure loads of traditional straight-fiber specimens.
\end{abstract}

\section{Introduction}

One of NASA's goals is to reduce the cost of air travel by $50 \%$ in the next 20 years. To achieve this goal, NASA has been involved in the development of the technologies needed for future low-cost, light-weight structures by varying the fiber orientations within a

\footnotetext{
*Senior AerospaceEngineer,MechanicsandDurability Branch, Associate Fellow,AIAA.

${ }^{+}$SeniorEngineer

${ }^{\dagger}$ Professor,Departments of Engineering Scienceand Mechanics, and

Aerospaceand OceanEngineering,AssociateFellow,AIAA
}

This material is declaredawork of the U.S. Govemmentand isnotsubjecttocopyight protection in theUnitedStates. composite structures for commercial transport aircraft. One such technology involves tailoring composite fibrous layer. Traditionally, fibrous layers of a composite laminate are arranged such that the orientation of the fibers within each layer is constant throughout a structural component, or only allowed discrete jumps from one section of the component to another. For structures possessing stress states that vary as a function of location within the laminate, not varying the fiber orientation angle to match the change in stress state results in inefficient utilization of the directional stiffness and strength characteristics of the fibrous composite laminates. Allowing the fibers to curve within the plane of the laminate furnishes a tailoring possibility to account for a non-uniform stress state, as well as providing other structural advantages such as the alteration of principal load paths. One of the traditional configurations in which stress distribution is highly non-uniform is the case of a flat panel with a central hole subjected to in-plane loading. Therefore, this sample problem will be studied to determine the effectiveness of stiffness tailoring through the use of curvilinear fiber paths to reduce stress concentrations around the hole and improve the overall load carrying capability of the structure.

One of the first approaches to improve compressive load carrying capability of composite laminates with an open hole was the experimental work of Chou, ${ }^{1}$ who inserted metal pins into woven fabric prior to curing, effectively pushing the fiber tows apart to create a molded hole. The resulting laminates possessed curvilinear fibers around the hole and exhibited improved open-hole strength compared to traditional straight-fiber laminates with drilled holes. Hyer and Charette $^{2}$ were among the first to investigate the influence of fiber orientation angle around a cutout for a flat plate with a circular hole. They proposed that improved designs could be realized by aligning the fibers with the principal directions of the stress field. Their finite element solution did demonstrate significant improvement with respect to material failure load levels; however their curvilinear fiber designs showed no increase in buckling load. A follow-up work by Hyer and Lee ${ }^{3}$ attempted to increase these buckling 
loads by using sensitivity analysis and a gradient search method to determine the optimal fiber orientation angles in different regions of the plate modeled by a grid of finite elements. This method produced designs with substantial increases in buckling loads, mostly through the mechanism of transferring the major stresses from the interior region to the edges of the panel. Nagendra et al. ${ }^{4}$ also used finite element analysis to develop manufacturable designs, and demonstrated comparable increases for plates with central holes.

Within the same time frame, research led by Gürdal and Olmedo $^{5,6}$ implemented a definition for a variable stiffness ply with continuously varying properties based on curvilinear fiber paths and formulated closed-form and numerical solutions for simple laminated plates. A follow-up design study by Waldhart et al. ${ }^{7}$ again indicated increased buckling performance due to the stiffness variation, which caused re-distribution of the internal loads toward the simply supported panel edges and introduced favorable transverse stresses that provided additional mechanisms to improve the buckling load of panels. This investigation also introduced several methods to define variable stiffness plies from a small set of parameters. The methods were based on a reference path defined by three angles, with subsequent paths being constructed either "parallel" to the original curved path or by defining paths identical to the reference curve but "shifted" in the direction perpendicular to the axis of fiber orientation angle variation. Considerations for the manufacturability of the plies, based on estimates for an advanced towplacement delivery system, were also included to ensure that the designs could be manufactured using these tow-steering methods.

The predictions based on these theoretical results prompted a need for experimental validation. Research by the present authors ${ }^{8}$ led to the fabrication of two panels using the VIPER ${ }^{\circledR}$ advanced tow-placement machine developed by Cincinnati Milacron." The panels were based on prototype "shifted" method designs from the study by Waldhart, ${ }^{7}$ and were built to validate the manufacturability of tow-steering through the application of the curvilinear-fiber formats. Photographs of the two panels during manufacture are shown in Fig. 1 and Fig. 2. The panel shown in Fig. 1 has individual tow drops that remove any overlap between successive paths of the ply, while the panel shown in Fig. 2 allows the tows to overlap to produce a panel with thickness build-ups that act as "integral stiffeners". Subsequent testing by $\mathrm{Wu}^{\mathbf{1 0 , 1 1}}$ confirmed the increased load-carrying capability of the variable stiffness panels with tow-drop and overlap construction, with gains of up to five times the compressive buckling load of a straight-fiber panel.
These results illustrate the potential of the process and, therefore, the need for a design tool that can integrate these curvilinear-fiber-ply formats with standard laminate design methodologies. Additional testing of laminates constructed with these curvilinear plies is also required to corroborate the earlier results and to further explore the possible benefits of the concept. Thus, the goal of the research presented in this paper is to design, manufacture, and test elastically tailored plates that exhibit improved performance compared to traditional fiber-reinforced laminates. The configuration considered herein is a rectangular flat panel with a central hole, loaded under axial compression. The elastic tailoring is accomplished through the variation of the fiber orientation angle within each ply, which can be manufactured with the aid of advanced tow placement machines. The design study, finite element modeling, and experimental results for this structure are presented.

\section{$\underline{\text { Laminate Design Utilizing Tow-Steered Plies }}$}

Common techniques for designing traditional composite laminates typically utilize the ply-orientation angles of the orthotropic layers as design variables. The objective of the design problem is to find the optimal stacking sequence that produces the best laminate, measured in terms of cost, weight, and performance. Optimization techniques for discretevalued design variables, such as genetic algorithms, ${ }^{12}$ are often used for this process, since laminates are typically manufactured using discrete ply-orientation angles. For example, ply angle choices are often limited to only $0^{\circ}, \pm 45^{\circ}$ and $90^{\circ}$ orientations to facilitate construction and reduce scrap. Furthermore, limiting a ply orientation to a set of well-chosen discrete angles, as opposed to treating them as continuous variables, enables the optimizer to investigate the global design space more efficiently, since stacking sequence design depends more on the number and ordering of the laminae rather then the exact values for the orientation angles.

For plies constructed using advanced tow-placement techniques, the manufacturability of a given ply angle is no longer limited to a small set of possibilities. These computer-controlled machines are capable of efficiently placing fiber-reinforced tows at almost any orientation desired while changing the orientation continuously from one location to another. The only major constraints in this process are the turning radius of the tow path (too small of a radius results in wrinkling of the tows) and the shape of the surface (the applicator head must have sufficient clearance for concave surfaces). However, this freedom is certainly a disadvantage for the optimizer, since the design space that was governed by simple discrete values for each 
ply now becomes a complex continuous variable domain which depends on spatially-varying orientation angles as well as practical manufacturing constraints. A remedy for this disadvantage is to use a parametric representation of the fiber orientation within the plate structure. Therefore, the following section will outline a useful parameterization for tow steered plies, which was originally proposed by Gürdal, ${ }^{5-8}$ that reduces the definition of a ply to a handful of global variables. These parameters can then be discretized into a tractable set to be used with a discrete-valued optimizer (much like the ply orientation angles for typical stacking sequence optimization), where the set of towsteered plies can be ensured to satisfy the manufacturing constraints before the design analyses are performed. That is, ensuring manufacturability reduces the design space by eliminating those combinations of the parameters from consideration. In the following sections, methods to analyze and design laminates that use these tow-steered definitions are discussed, followed by design results for the sample problem of a panel with a central hole.

\section{Parameterization of Tow-Steered Plies}

The design parameters used here are based on earlier work by Gürdal, ${ }^{5-8}$ who introduced a general reference path based on a linear variation of the fiber orientation angle in a specified direction. The method assumes that the surface to be considered can be represented by twodimensional surface coordinates $(x, y)$, and is based on the specification of the paths traveled by the head of the tow-placement machine during fabrication.

A graphical representation of the reference path definition is shown in Fig. 3. The origin and major direction of the reference path are defined by a point $\left(x_{0}, y_{0}\right)$ and angle $\phi$, respectively. The relative translation and rotation of a tow-steered ply definition can be easily applied. At the origin, the angle $T_{0}$ represents the initial orientation of the tows with respect to the major direction of variation. The orientation angle varies linearly in a saw-tooth manner such that its value is $T_{1}$ at a distance $d$ along the variation axis, and then changes back to $T_{0}$ at a distance of $2 d$. The variation is symmetric about the origin of variation. This linear variation of the orientation angle yields a smooth continuous curve which represents the path of the centerline of the tow placement machine's applicator head (the dotted line in Fig. 3). For the VIPER $^{\circledR}$ tow-placement machine used here for fabrication, the applicator head has a total width of 3 inches ( 24 individual tows, each 1/8 in. wide), resulting in a swath of material being laid down in the manner shown in Fig. 3.

To construct an entire ply using this reference curve, additional courses are laid down by shifting the reference path in a direction perpendicular to the $\phi$-direction. This shift distance is calculated so that no gaps exist between adjacent courses. Due to the curvature of the reference path and the shifted construction method used for additional courses, overlaps may exist between tows of adjacent courses (as shown in Fig. 4, in which the light gray area represents the overlap region). Two techniques are employed to handle this overlap. The first, referred to as the "tow-drop" method, instructs the fiber placement machine to cut/drop individual tows within each pass so that no thickness build-up occurs in the regions of overlapping paths. These fiber terminations result in constant thickness panels that contain small wedge-like areas that contain no fibers due to the dropping of the individual tows. The second construction method is called the "overlap" method and allows for full overlap between adjacent paths, which leads to considerable thickness build-ups for some areas of the panel (as is evident for the overlap panel shown in Fig. 2). Numerical algorithms were developed to generate course data for a given ply-path definition and construction method as well as to find the actual fiber orientation angle for a given $(x, y)$ point (note that for the overlap method, some locations may have two angles due to the overlap of neighboring paths).

For the present design study, the origin $\left(x_{0}, y_{0}\right)$, major direction $\phi$, and characteristic distance $d$ are determined by the geometry and expected loading of the structure, and the angles $T_{0}$ and $T_{1}$ are the design variables. Such a ply is designated by the shorthand notation $\phi<T_{0} \mid T_{1}>$. Additionally, a calculation of the turning radius along the path can be easily performed once all the parameters are defined, and configurations that possess a minimum turning radius below the allowable limit (25 in. for the $\operatorname{VIPER}^{\circledR}$ tow-placement machine) can be removed from the allowable set of designs. Further details on parameter restrictions are discussed in the formulation of the design study.

\section{Analysis of Tow Steered Laminates}

Due to the variable nature of the fiber orientation for a tow-steered ply, each location has a different stacking sequence that depends on the parameter specification. This variation leads to laminates with spatially-varying stiffness measures, which require modifications to standard analysis techniques. Two such techniques used to assess the performance of tow steered laminates are described in this section.

Finite Element Solutions. For a traditional finite element package, the variable stiffness behavior implies that each element must have a unique stacking sequence associated with it and that the stiffness parameters are constant over the domain of the element. The nature of 
the finite element technique also requires that the smooth curves of the tow paths will be discretized by the geometry of the element mesh, and therefore a dense mesh is needed for accurate modeling of the stiffness variation. In general, this mesh requirement leads to large models that require an automatic algorithm to calculate each stacking sequence and link it to the corresponding element location. To facilitate this re-definition, software was developed that can transform suitable finite element meshes into variablestiffness laminates that are specified using the towsteering parameters introduced earlier.

A screenshot of the re-definition software, referred to as $L D T$ for Laminate Definition Tool, is displayed in Fig. 5. The necessary parameters for the tow-steered laminate definition are entered in the appropriate fields. To perform the transformation, a valid finite element model is selected for the input file name. Presently, models are limited to flat structures lying in the $x-y$ plane, though in general any structure that can be represented by surface coordinates can be implemented. The input file must be in an acceptable format for the finite element package that is being used for analysis (GENESIS/NASTRAN $^{13}$ and STAGS ${ }^{14}$ are currently supported). Subsequent execution reads in the file, calculates a stacking sequence at the centroid of each element and assigns it to a unique property, and rewrites the input file with the elements associated with the new properties. Though the procedure is quite straightforward, the large number of stacking sequence calculations and associated input/output leads to significant process time. For example, finite element models that were used in this study, consisting of 10,800 elements, required approximately five minutes to be transformed into tow steered designs. Though this is a reasonable allowance for the generation of new models, it is generally prohibitive within a design process. Moreover, the computational cost of each finite element anlaysis is high, making it undesirable for the repetitive analyses that are needed for conceptual or preliminary design studies. Therefore, a more efficient Rayleigh-Ritz technique that solves the underlying governing equations for the variable stiffness laminates has been developed for simple flat plates. While the Rayleigh-Ritz technique is very useful for initial design work, it is not a substitute for the more detailed finite element analysis needed for final design.

Rayleigh-Ritz Solution. This numerical solution concerns a flat rectangular panel with simply-supported edges subject to combined in-plane loading. The panel is constructed of a symmetric laminate using traditional and curvilinear fiber-reinforced plies. For variablestiffness laminates, algorithms exist to calculate the Classical Lamination Theory (CLT) stiffness terms $\left(A_{\mathrm{ij}}\right.$,
$B_{\mathrm{ij}}, D_{\mathrm{ij}}$ ), which are continuous functions of the panel coordinates, at an arbitrary point based on the towsteered layup definitions described earlier. For the inplane response, the loading is introduced through constant normal and shear displacements at the edges and the reaction forces are summed to calculate the average stress resultant applied to each edge. This technique is used to allow for stiffness variations within the panel that generate non-constant distributions of stresses along the boundaries. Following the RayleighRitz technique, typical expansions in terms of double trigonometric series are formulated for the in-plane displacements $u$ and $v$ as well as the variable CLT stiffness parameters. Insertion of these expansions into the integral representing the total potential energy of the system and minimizing with respect to the unknown expansion coefficients yields a linear system of equations. For constant-stiffness laminates, the equations reduce to the trivial solution of constant loading. The in-plane solution for the variable stiffness laminates is used to measure material failure by investigating the stress state for an evenly spaced grid of points within the panel. Additionally, a linear buckling estimate is performed, where the stress resultants serve as the prebuckling state and the out-ofplane displacement $w$ and bending stiffness matrix $D_{i j}$ are similarly expanded in trigonometric series and inserted into integrals for the potential energy of the panel.

The solution just described is formulated as a Fortran 90 code. Though both the tow-drop and overlap methods are implemented, the discontinuities in thickness due to the overlap technique are not as accurately modeled through the trigonometric expansions, and require significantly more terms (and therefore more computation time) than constant thickness laminates for accurate modeling. Thus most of the computations performed with this algorithm assume no overlaps are present. Computation times for a typical variable stiffness solution (using seven terms in each direction for the expansions of the displacements) average around three seconds on a typical 400-MHz PC platform. For traditional straightfiber laminates, which possess constant stiffness throughout the panel, several simplifications can be made in the equations and the analysis times reduce to significantly less than half a second. Comparisons of the results of this numerical solution have been made with dense finite element models, and have been shown to agree well within tolerable limits (less than $1 \%$ error) for both the in-plane response and buckling estimate of a tow-drop design. ${ }^{15}$ 


\section{Design Study for Flat Panel with Central Hole}

To assess the potential of the tow-steering concept to increase buckling loads, a design study is conducted for a flat panel with a central hole under axial compression. Panel dimensions, boundary conditions, and hole sizes are shown in. Fig. 6. The presence of the central hole and the fixed boundary conditions at the top and bottom lead to a highly variable stress state within the structure, for which the curvilinear-fiber formats exhibit promise in increasing buckling and failure loads. A design study is performed to assess the increased buckling load that is attainable using tow-steered plies.

Design Technique. Since the finite element model definition and analysis for tow-steered laminates are computationally expensive, stacking-sequence-design optimization that utilizes a discrete mathematical programming approach is not suitable due to the large number of analysis runs required. Therefore, the simpler Rayleigh-Ritz solution is used for the preliminary design phase. Though this approach will enable a thorough investigation of the design space, the simplified analysis does not take into account the central hole, the test fixture boundary conditions, or allow for calculations representing the overlap method. However, earlier results ${ }^{7}$ have shown that the major mechanism to improve the buckling load using towsteered plies is to redistribute the load away from the center of the panel by having stiffer sections near the edges. Since the tow-drop method can fulfill this objective, the simplified analysis for a panel with identical dimensions as the test specimen is used in the preliminary design phase. The results will supply several candidate designs that will be further analyzed with the more accurate finite element techniques. The prototype designs that will be manufactured and tested are determined. from this set

Design Study Results. The design studies were conducted using $O L G A^{16,17}$ (Optimization of Laminates using Genetic Algorithms), an in-house laminate design tool that implements tow steered plies for flat panel structures. Two design studies were conducted. The first design study considered only straight-fiber plies, to serve as a baseline for the tow-steered panels. A 15 in. by 20 in. rectangular panel constructed from AS4/977-3 material was defined within $O L G A$, subject to a $1 \mathrm{lb} / \mathrm{in}$. compressive load in the axial direction. Material properties corresponding to a typical AS4/9773 system were used in the $O L G A$ and finite element design studies. Specifically, longitudinal modulus, $\mathrm{E}_{1}=18.83$ Msi, transverse modulus, $\mathrm{E}_{2}=1.34 \mathrm{Msi}$, shear modulus $\mathrm{G}_{12}=0.64 \mathrm{Msi}$, Poisson's ratio $v_{12}=0.36$, density $\rho=0.058 \mathrm{lb} / \mathrm{in}^{3}$ and ply thickness of 0.0075 inches.
Loads corresponding to buckling and to material failure using the Tsai-Hill criterion were calculated for the candidate laminates. Laminates for fabrication were required to have greater failure loads than buckling loads. The laminate was further restricted to be symmetric and constructed of twenty plies. The design variables were based on the fiber-orientation angles $T_{0}$ and $T_{1}$ within each layer and were allowed to vary in $15^{\circ}$ increments. Two-ply stacks of plus/minus orientations were also used to ensure the laminate was balanced and to effectively reduce the size of the design space. The best design from the straight-fiber study was found to be a laminate with a stacking sequence of $\left[ \pm 45_{2} / \pm 30 / \pm 45 / \pm 15\right]_{\mathrm{s}}$, which possessed a buckling load of $7903 \mathrm{lbs}$.

The second design study used the tow-drop method for the internal plies. The external plies were constrained to have straight fibers so that gaps and overlaps did not exist on the exterior of the laminate. The origin of the variation was placed in the center of the panel, the variation direction was perpendicular to the loading $(\phi=0)$, and the value of characteristic dimension $d$ was chosen to be one-half the width of the panel. The buckling predictions for the five best tow-drop designs are shown in Table 1.

Table 1. Results from $O L G A$ design study

\begin{tabular}{|c|c|}
\hline Design Number and Layup & $\begin{array}{l}\text { Buckling } \\
\text { load (lbs) }\end{array}$ \\
\hline 0 (Baseline): $\left[ \pm 45_{2} / \pm 30 / \pm 45 / \pm 15\right]_{\mathrm{s}}$ & 7,903 \\
\hline $1:\left[ \pm 45 / 0 \pm<45\left|60>_{2} / 0 \pm<45\right| 30>/ 0 \pm<45 \mid 15>\right]_{\mathrm{s}}$ & 9,313 \\
\hline 2: $\left[ \pm 45 / 0 \pm<45|60>/ 0 \pm<30| 15>/ 0 \pm<45 \mid 60>_{2}\right]_{\mathrm{s}}$ & 9,244 \\
\hline 3: $\left[ \pm 45_{2} / 0 \pm<45|60>/ 0 \pm<30| 15>/ 0 \pm<45 \mid 60>\right]_{\mathrm{s}}$ & 9,225 \\
\hline $4:\left[ \pm 45 / 0 \pm<45\left|60>_{2} / 0 \pm<30\right| 15>/ 0 \pm<45 \mid 60>\right]_{\mathrm{s}}$ & 9,211 \\
\hline $5:\left[ \pm 45 / 0 \pm<30|45>/ 0 \pm<45| 60>_{2} / 0 \pm<30 \mid 15>\right]_{\mathrm{s}}$ & 9,186 \\
\hline
\end{tabular}

To verify the trends found using $O L G A$, five candidate designs were then analyzed with STAGS,${ }^{14}$ using both a small (1.5-in. diameter) and large (3.0-in. diameter) hole. The finite element models for the rectangular panel with a small or large central hole are shown in Fig. 7 and Fig. 8, respectively. The small-hole model contains 6206 elements with $\sim 37,000$ degrees of freedom, while the large-hole model has 5694 elements and $\sim 34,500$ degrees of freedom. These models are designed to accurately model the test fixture to be used. On the top and bottom edges, the two inches of potting is modeled by allowing displacement only in the axial direction. The load is introduced through a constant compressive force at the top of the potted edge, which is constrained to remain straight, while the bottom edge is held stationary. Out-of-plane displacement and 
rotation about the $x$-axis are restrained approximately 0.2 inches from each unloaded edge to simulate the effect of the knife edges in the test. The mesh density yields elements that are approximately $0.25 \mathrm{in}$. on each side, which was determined through parameter studies to be small enough to accurately model the variations in thickness due to the curvilinear fibers and the overlaps.

Predictions of the buckling loads for the baseline design (0) and the tow-steered designs (1-5) using both construction techniques and based on finite element analysis are shown in Table 2.

Table 2. Finite element results for candidate designs

\begin{tabular}{|c|c|c|c|c|}
\hline \multirow{2}{*}{$\begin{array}{l}\text { Design } \\
\text { Number }\end{array}$} & \multicolumn{4}{|c|}{ Buckling load (lbs) } \\
\hline & $\begin{array}{c}1.5 \text {-in. } \\
\text { hole }\end{array}$ & $\begin{array}{c}3.0 \text {-in. } \\
\text { hole }\end{array}$ & $\begin{array}{c}1.5 \text {-in. } \\
\text { hole }\end{array}$ & $\begin{array}{c}3.0-\text { in. } \\
\text { hole }\end{array}$ \\
\hline \multirow[t]{2}{*}{0} & 9,124 & 8,339 & - & - \\
\hline & \multicolumn{2}{|c|}{ Tow-drop Method } & \multicolumn{2}{|c|}{ Overlap Method } \\
\hline 1 & 10,168 & 9,388 & 13,653 & 12,710 \\
\hline 2 & 9,988 & 9,141 & 13,787 & 12,954 \\
\hline 3 & 9,904 & 9,096 & 12,530 & 11,725 \\
\hline 4 & 10,387 & $\mathbf{9 , 5 5 7}$ & 14,301 & 13,499 \\
\hline 5 & 10,143 & 9,306 & 13,541 & 12,738 \\
\hline
\end{tabular}

Differences between the buckling loads presented in tables 1 and 2 (OLGA predictions and STAGS predictions, respectively), are attributed to the presence of the central holes as well as the fixed-end boundary conditions. From the results of Table 2, the fourth design was chosen as the prototype design to be used for manufacture and testing and is identified by bold type in Tables 1 and 2.

\section{Manufacturing of Prototype Designs}

The chosen layup for the tow-steered design, denoted as $\left[ \pm 45 / 0 \pm<45\left|60>_{2} / 0 \pm<30\right| 15>/ 0 \pm<45 \mid 60>\right]_{s}$, must be translated into ply data that can be decoded by the towplacement machine. Algorithms were produced to perform this task, which at the same time served as a useful tool for visualizing the construction of the ply and improving the design of the laminate. The next two sections discuss alterations that were made to the prototype designs before fabrication to improve the reliability of the test panels. However, due to unavailability of the planned material, a G40-800/977-2 material system was substituted. The primary difference between these two systems is the tape thickness. The original design was for tape with a 0.0075-inch thickness but the new material has a tape thickness of 0.0055 inches. Therefore, even though the design stacking sequences were used, the final panels are much thinner and therefore have lower buckling loads and additional analysis was required to correlate analysis and test results.

\section{Staggering Technique for Tow-Steered Plies}

The prototype tow-steered layup contains two different steered-ply groups: a $0 \pm<45 \mid 60>$ group, of which there are three pairs; and a single pair of $0 \pm<30 \mid 15>$. After calculating the resulting ply paths for each ply and analytically assembling the entire laminate, areas of thickness build-up for the overlap method can be plotted. Such a result is shown in Fig. 9, where the central holes to be machined are shown as dashed circles and the shaded regions represent an increased number of plies due to the overlaps between tow courses. However, though the increased plies provide greater stiffness near the unloaded edges of the panel (which is what increases buckling loads), the discrete increase from 20 plies to 26 plies in the major overlap regions is considered to be too drastic to reliably manufacture. Therefore, the ability to easily change the origin of the tow-steered design, through the use of the $\left(x_{0}, y_{0}\right)$ parameters, was called upon to stagger the $0 \pm<45 \mid 60>$ ply groups in the vertical direction and provide a more consistent thickness variation for the panel. Note that this staggering is different from the shifting of the tows discussed previously. The shifting in the reference path is to account for the non-zero width of the tow and is applied to each tow within a ply. The staggering is to smooth the overall thickness and changes the origin of the entire ply. This modified layup, shown in Fig. 10, has the same stacking sequence as the one in Fig. 9 but because of staggering it has an improved thickness variation as can be observed from the figure. This alteration was also performed for the tow-drop method to spread out the locations of the gaps resulting for the tow drops. Although the interweaving reduced the variation in thickness, the buckling load calculated by finite element analysis showed no appreciable decrease. The resulting changes in buckling load were less than $1 \%$. Therefore, this technique of interweaving ply groups is recommended whenever identical ply groups are present in a tow-steered design.

\section{Fabrication and Preparation of Test Specimens}

In order to minimize the set-up time and cost of fabrication, four test panels with the same layup were constructed simultaneously as one large sheet and then individual specimens machined after cure. Simultaneous fabrication also reduces overall machine time and eliminates scrap as the edges always have unusable tow-start areas, as well as producing test specimens that are manufactured and cured in the same environment. 
The formulation used to define the curvilinear fiber courses is conducive to defining the layup for the large sheet, since the definition repeats with period $2 d$ (which is equal to the individual panel width by design). The pattern for cutting four test specimens from one large panel is shown in Fig. 11 for the overlap method. The pattern in the figure shows the thicknesses without staggering the plies and is for illustrative purposes only. Staggering was used in the fabricated panel. In the figure, the darker regions indicate the increased thickness build-up due to the three $0 \pm<45 \mid 60>$ ply groups and the $0 \pm<30 \mid 15>$ ply group is the lighter and shallower curves. Note, however, that the overlap regions are symmetric about each panel center due to the cyclic nature of the tow steering definition, yet the intersections of oppositely oriented overlap regions are not regularly spaced across the sheet. This artifact is due to the fact that the locations of the intersections do not depend merely on $\mathrm{d}$, the characteristic distance of the fiber orientation definition, but also on the direction of the fiber course and the width of the tow-placement head. For example, if the general direction of the overlap regions are aligned at positive and negative forty-five degrees, the intersections will occur much more frequently than if the direction is at fifteen degrees. Therefore, when overlaps are used, the four specimens are not exactly identical due to the nonregular spacing of the intersection points.

Finally, in light of the result that the overlap panels depend on the width of the tow placement head, an investigation was conducted to determine the best head width to use for the tow steered designs. The default value corresponds to 24 tows that are $1 / 8$ inch wide (3.03 inches total, allowing for a $1 \%$ space between tows). Models were constructed and analyzed using 12 to 36 tows to determine if the different head widths demonstrated increased buckling resistance due to the different location of the overlap intersections. However, the results showed little difference between designs with different head widths (less then $2 \%$ over the total range). Therefore the designs will use the default value of 24 tows, which is the maximum number of tows in the actual tow placement machine and should minimize fabrication time.

Also shown in Fig. 11 are the locations of the central holes for testing, which will be machined in when the specimens are processed. For the tow-steered panels (manufactured using the tow-drop and overlap methods), two test specimens each for the two different hole sizes were produced. For the straight-fiber panel, a baseline specimen with no hole was produced, as well as one with the smaller central hole and two with the larger hole. The test specimens are labeled and differentiated by construction method ( $\mathrm{A}=$ straight- fiber; $\mathrm{B}=$ tow-drop; $\mathrm{C}=$ overlap) and position on the large panel (1-4, numbered from left to right).

All specimens were potted in two inches of epoxy compound on each end and the ends ground flat and parallel to each other. A photograph of an overlap specimen with a large hole (specimen C3) is shown in Fig. 12. Knife-edge supports were applied approximately 0.2 inches from the unloaded edges as shown in Fig 6. to restrain out-of-plane motion. The specimen was instrumented with strain gages and DCDTs (Direct Current Displacement Transducers). Back-to-back strain gages were applied at the midlength location and 2 inches from the potting for each specimen, as shown in Fig. 12. Two DCDTs were used to measure end-shortening and two were used to measure out-of-plane displacement for each specimen. An out-of-plane displacement measurement was taken at the axial quarter point directly above the center of the specimen and another was taken close to the hole, at the mid-length location. Measurement locations are shown in Fig. 6. Shadow Moiré interferometery was also used to monitor out-of-plane displacement patterns of each specimen. Each specimen was loaded to failure at a rate of approximately 1,000 lb/min.

\section{$\underline{\text { Results and Discussion }}$}

Buckling load predicted by finite element analysis using models conforming to the manufactured designs, using the smaller thicknesses of 0.0055 inches per ply for the as-manufactured panels, are shown in Fig. 13. Experimentally determined buckling and failure loads are also shown in Fig. 13. All specimens carried load well in excess of the buckling load. The overlap specimens carried significantly more load than the comparable tow-drop specimens.

Displacement predictions are shown in Fig. 14-16 for straight-fiber, tow-drop, and overlap specimens with large holes (specimens A4, B3, and C3, respectively). Experimental results are also shown in the figures. Reasonable agreement is observed for the straight-fiber specimen but buckling and post-buckling behavior is not as accurately predicted for the tow-steered specimens. However, the tow-drop specimens generally have buckling loads about 10 percent greater than the comparable straight-fiber specimens, while the overlap specimens achieve buckling loads almost double that of the constant-stiffness counterparts. Finite element analysis accurately predicts mode shapes and mode shape changes in most cases, as evidenced by the reversal of displacement shown in figures 14-16 for both the experiment and the analysis. Experimental buckling loads are significantly greater than analytical predictions for some cases. Possible explanations for this difference include the differences in the assumed and actual material properties, thermal effects involved 
in curing anisotropic panels, and imperfections caused by manufacturing irregularities. Since these factors would contribute significantly to a failure prediction, no analytical predictions of failure are presented and analysis was terminated in some cases at load levels less than the experimental failure load, but always continued until at least double the initial buckling load.

Out-of-plane displacement patterns reveal that most specimens initially buckled into one half-wave in each direction. Some specimens changed mode shape by consequently buckling into two half-waves in the axial direction. Photographs of out-of-plane displacement patterns for the large hole specimens are shown in Fig. 17-18 (straight-fiber specimen A4), Fig. 19-20 (towdrop panel B3), and Fig. 21-22 (overlap panel C3) for load levels corresponding to a load just greater than the initial buckling load and for a load just less than failure. No predictions for failure loads are made. However, as shown in Fig. 13, specimen failure loads are significantly greater than buckling loads in all cases with the overlap specimens carrying loads more than three times their buckling loads. Most specimen failures were in the corners, not at the hole edge.

\section{Concluding Remarks}

Software tools developed for the analysis and design of tow-steered composite laminates were used to explore the benefits of curvilinear fiber concepts for tailoring of rectangular laminates with circular holes. Parameterization of tow-steered plies using a small number of variables provided an efficient strategy for rigorous optimization based on a simplified analysis procedure. Software tools also made it possible to integrate the tow-steered ply definitions with a state-ofthe-art finite element program for detailed analysis of the laminates under general loading and boundary conditions representative of experimental conditions.

The systematic procedure from design to fabrication employed in this project produced an excellent method for incorporating manufacturing constraints within the design process. By limiting the design choices to feasible configurations, the optimized designs could be directly translated to machine language to provide exact agreement with the prototype parts. The results presented here indicate that significant increases in load-carrying capability compared to traditional straight-fiber laminates can be realized, and demonstrate that elastic tailoring is a useful technique for improving the performance of composite laminates. Experimental results verify that the tow steering technique can be used to fabricate specimens with increased buckling and failure loads. To achieve a more accurate analytical results for load beyond the buckling load for these specimens, a more detailed finite element analysis taking into account more of the manufacturing irregularities in the fabricated pieces would be required.

\section{$\underline{\text { References }}$}

1. Yau, S. S., and Chou, T. W., "Strength of WovenFabric Composites with Drilled and Molded Holes," Composite Materials Testing and Design, ASTM STP972, American Society for Testing and Materials, Philadelphia, PA, 1988, pp. 423-437.

2. Hyer, M. W., and Charette, R. F., "The Use of Curvilinear Fiber Format in Composite Structure Design," Proceedings of the $30^{\mathrm{th}}$ AIAA/ASME/ASCE/AHS/ASC Structures, Structural Dynamics and Materials (SDM) Conference, New York, NY, 1989, paper no 1404.

3. Hyer, M. W., and Lee, H. H., "The Use of Curvilinear Fiber Format to Improve Buckling Resistance of Composite Plates with Central Holes," Composite Structures, Vol. 18, 1991.

4. Nagendra, S., Kodiyalam, A., Davis, J. E., and Parthasarathy, V. N., "Optimization of Tow Fiber Paths for Composite Design," Proceedings of the $36^{\text {th }}$ AIAA/ASME/ASCE/AHS/ASC Structures, Structural Dynamics and Materials (SDM) Conference, New Orleans, LA, April 1995, AIAA paper no. 1275.

5. Gürdal, Z., and Olmedo, R., "In-Plane Response of Laminates with Spatially Varying Fiber Orientations: Variable Stiffness Concept," AIAA Journal, Vol. 31, (4), April 1993.

6. Olmedo, R., and Gürdal, Z., "Buckling Response of Laminates with Spatially Varying Fiber Orientations," Proceedings of the $34^{\mathrm{th}}$ AIAA/ASME/ASCE/AHS/ASC Structures, Structural Dynamics and Materials (SDM) Conference, La Jolla, CA, April 1993, paper no. 1567.

7. Waldhart, C. J., Gürdal, Z., and Ribbens, C., "Analysis of Tow Placed, Parallel Fiber, Variable Stiffness Laminates," Proceedings of the $37^{\text {th }}$ AIAA/ASME/ASCE/AHS/ASC Structures, Structural Dynamics and Materials (SDM) Conference, Salt Lake City, UT, April 1996, paper no. 1569.

8. Tatting, B. F., and Gürdal, Z., "Design and Manufacture of Tow-Placed Variable Stiffness Composite Laminates with Manufacturing Considerations," Proceedings of the $13^{\text {th }}$ U.S. National Congress of Applied Mechanics (USNCAM), Gainesville, FL, 1998.

9. Evans, D. O., Vaniglia, M. M., and Hopkins, P. C., "Fiber Placement Process Study," $34^{\text {th }}$ International SAMPE Symposium, May 1989. 
10. Wu, K. C., and Gürdal, Z., "Thermal Testing of Tow-Placed Variable Stiffness Panels," Proceedings of the $42^{\text {nd }}$ AIAA/ASME/ASCE/AHS/ASC Structures, Structural Dynamics and Materials (SDM) Conference, Seattle, WA, April 2001, paper no. 1190.

11. Wu, K. C., Gürdal, Z., and Starnes, J. H., "Buckling and Postbuckling of Tow-Placed Variable Stiffness Panels," Proceedings of the $43^{\text {rd }}$ AIAA/ASME/ASCE/AHS/ASC Structures, Structural Dynamics and Materials (SDM) Conference, Denver, CO, April 2002, paper no 1512.

12. Gürdal, Z., Haftka, R.T., and Hajela, P., Design and Optimization of Laminated Composite Materials, John Wiley and Sons, Inc., New York, NY, 1999, pp. 185-218.

13. Vanderplaats Research and Development, Inc., "GENESIS User Manual," Colorado Springs, CO, 2000.

14. Rankin, C. C., Brogan, F. A., Loden, W. A., and Cabiness, H. D., "STAGS Users Manual," Lockheed Martin Missiles \& Space Co., Inc., Report LMSC P032594, June 2000.

15. Tatting, B., and Gurdal, Z., Design and Manufacturing of Elastically Tailored Tow Placed Plates, NASA/CR 2002-211919, August 2002.

16. Soremekun, G. A. E., and Gürdal, Z., "Advanced Genetic Algorithms for Designing Complex Laminated Structures," Proceedings of the American Helicopter Society Hampton Roads Chapter, Structure Specialists' Meeting, Williamsburg, VA, October 2001.

17. Tatting, B. F., and Gürdal, Z., "Analysis and Design of Tow-Steered Variable Stiffness Composite Laminates," Proceedings of the American Helicopter Society Hampton Roads Chapter, Structure Specialists' Meeting, Williamsburg, VA, October 2001.

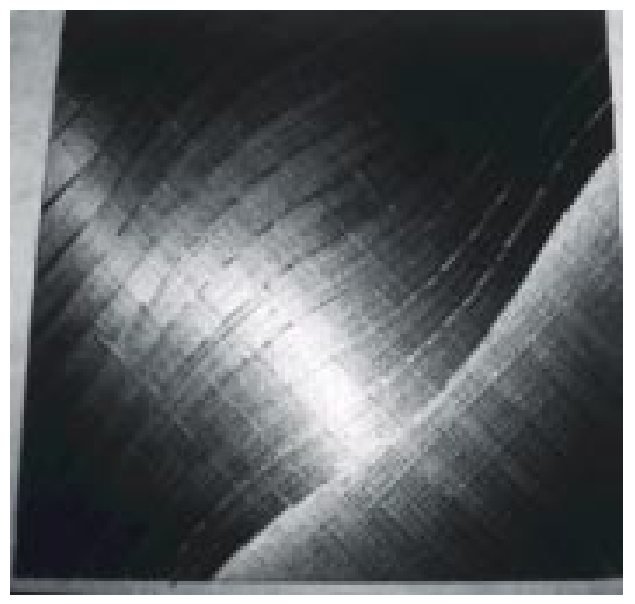

Fig. 1. Fabricated panel using tow-drop method.

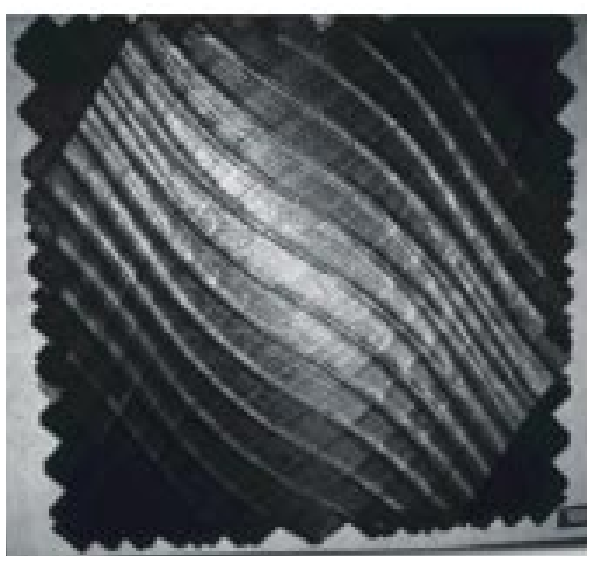

Fig. 2. Fabricated panel using overlap method. 


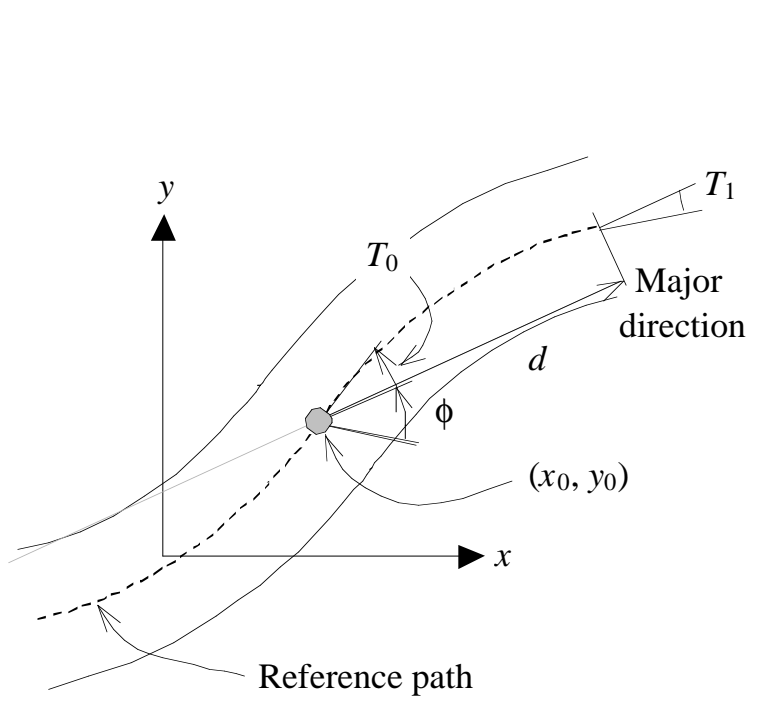

Fig. 3. Geometry of reference path.

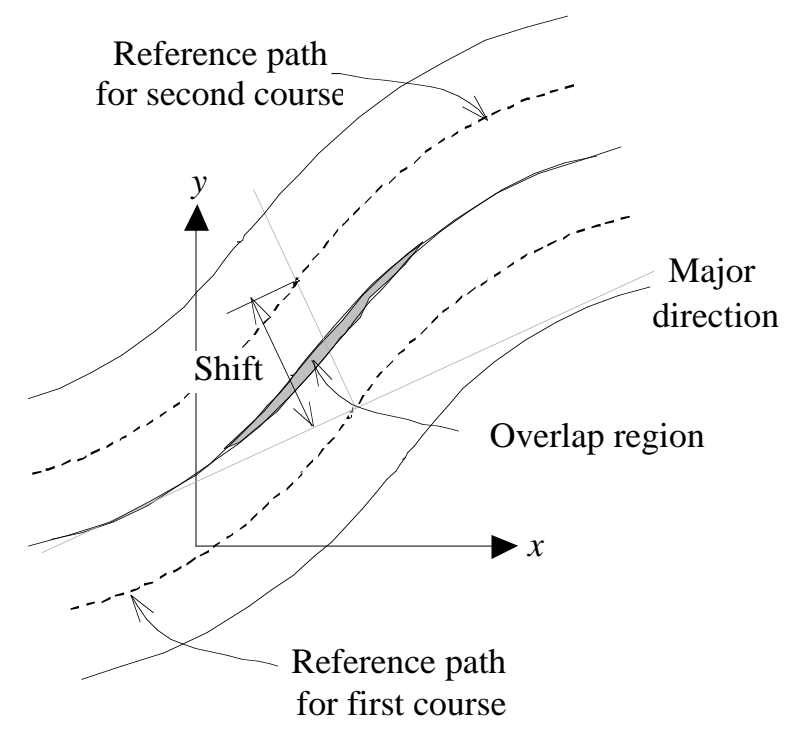

Fig. 4. Adjacent courses with overlap region.

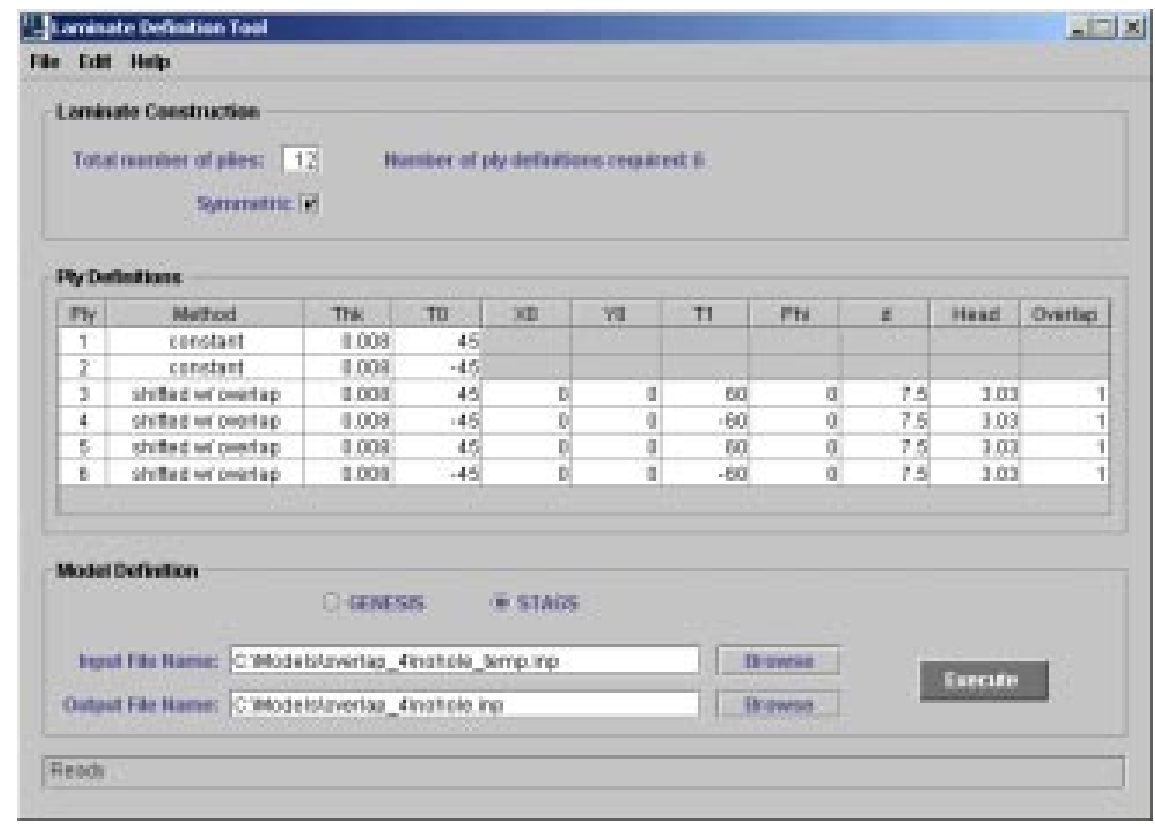

Fig. 5. Screenshot of Laminate Definition Tool (LDT). 


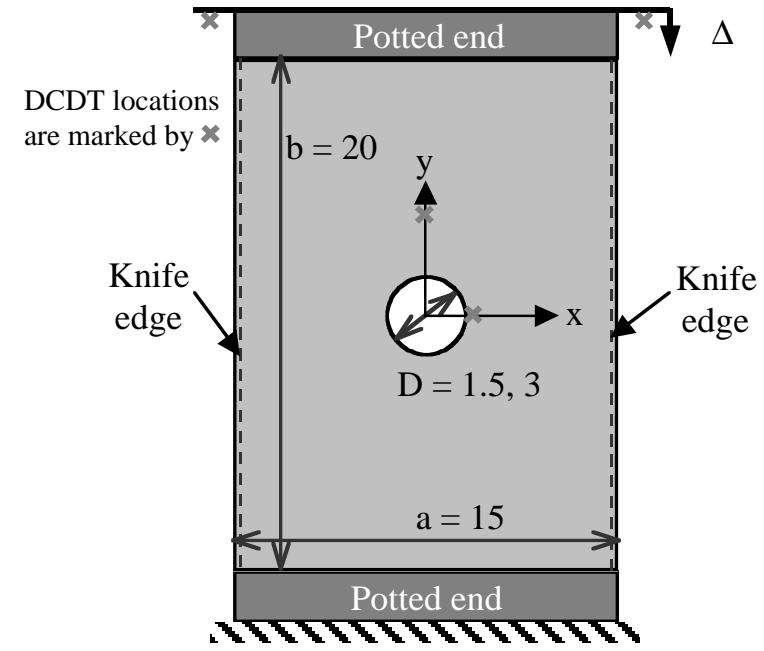

Fig. 6. Geometry of test specimen (lengths in inches).

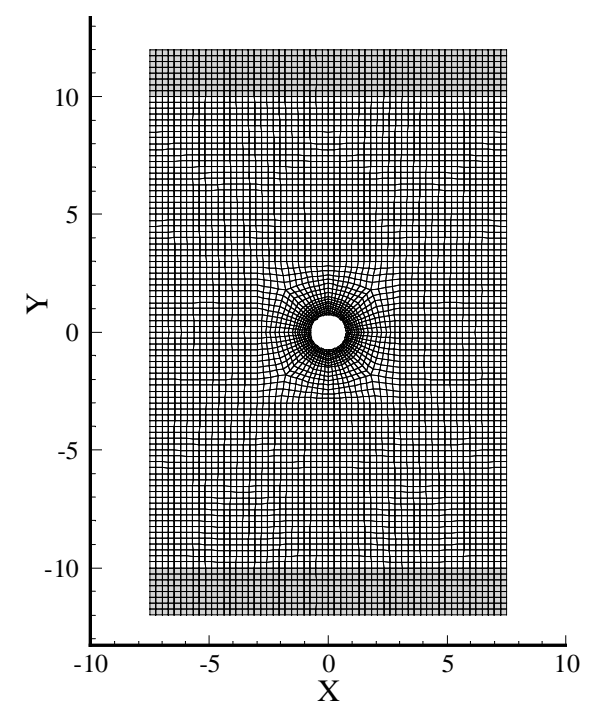

Fig. 7. Finite element mesh for small-hole model.

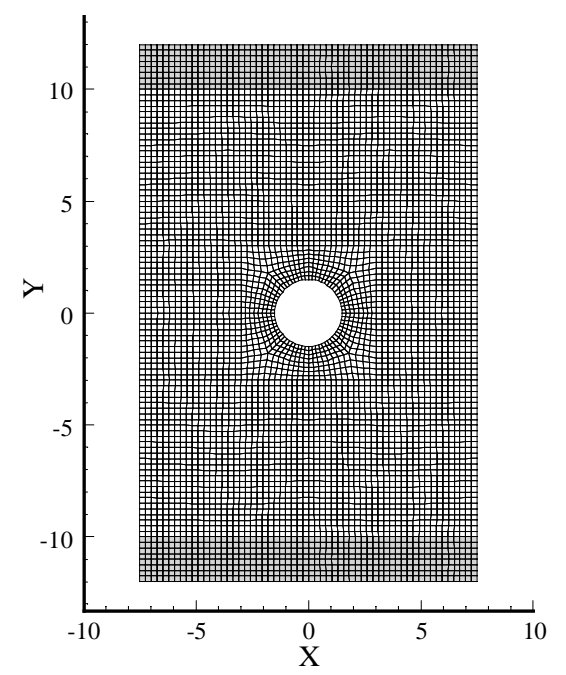

Fig. 8. Finite element mesh for large-hole model.

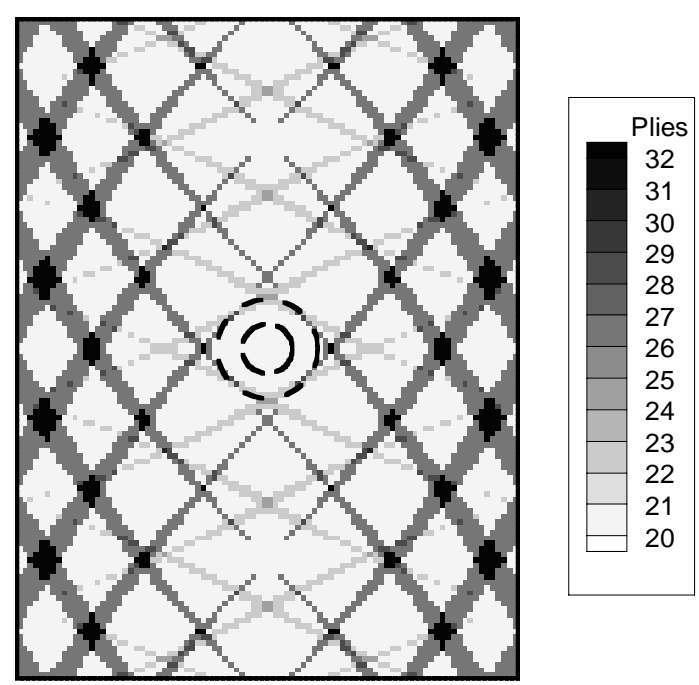

Fig. 9. Thickness distribution for original overlap model without staggering plies.

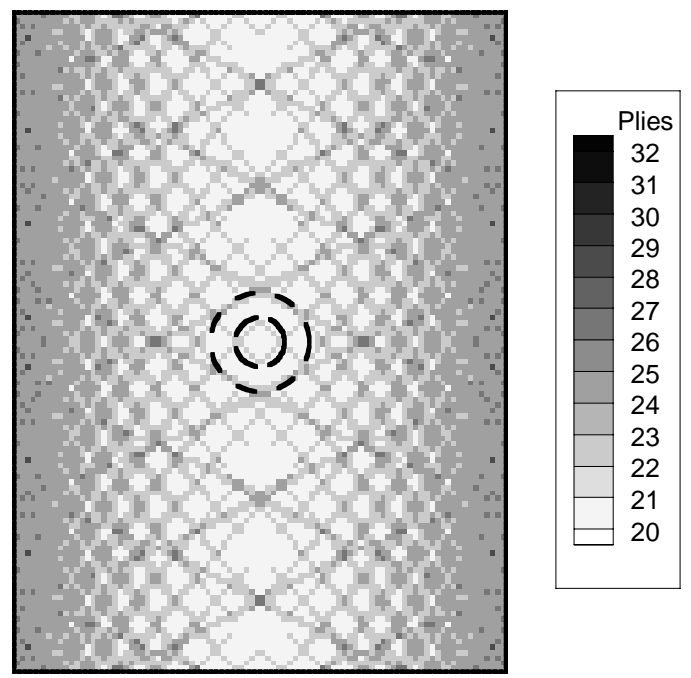

Fig. 10. Thickness distribution for modified overlap model with staggered plies. 


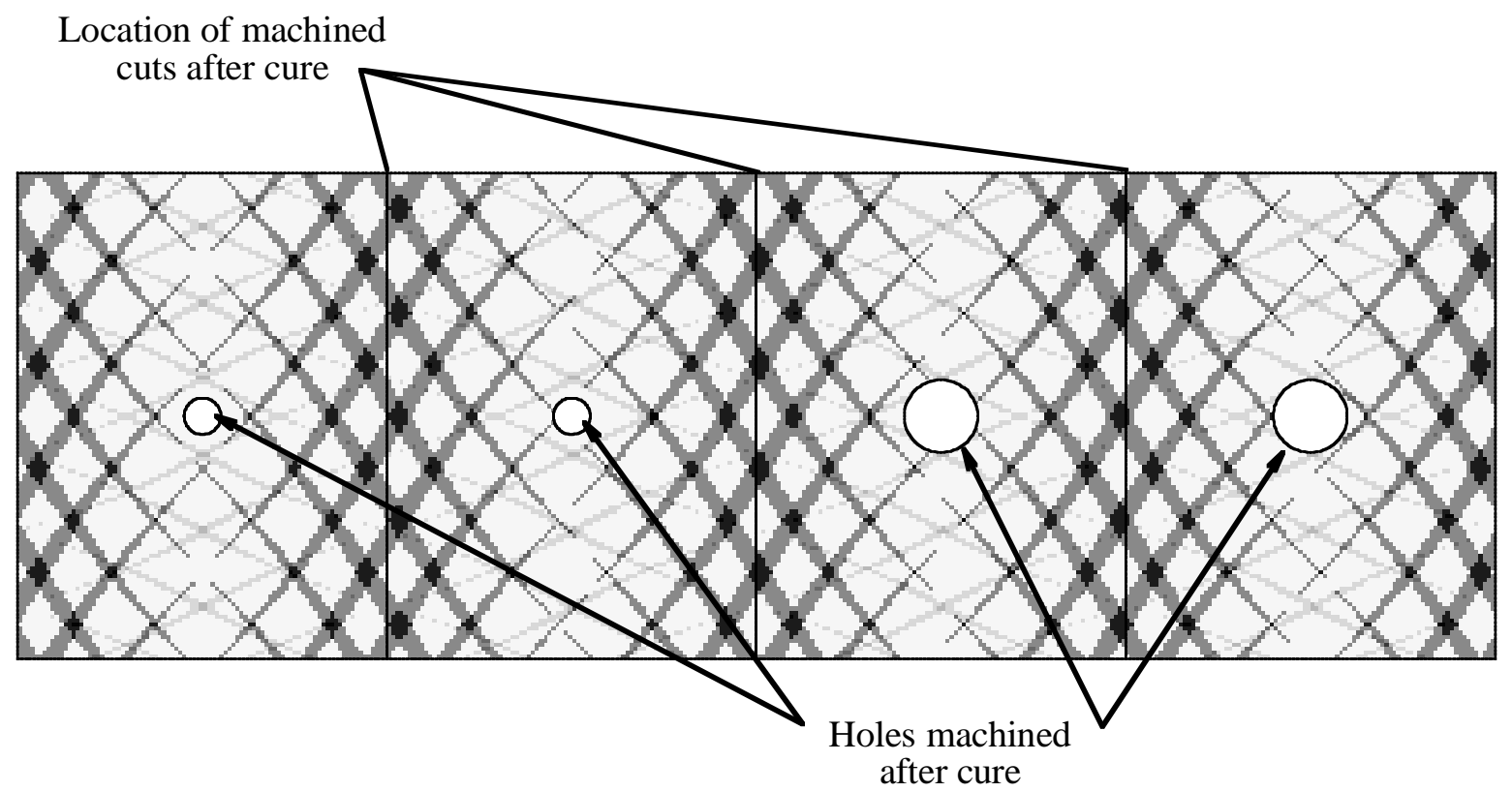

Fig. 11. Panel construction of adjacent specimens. Note that the fabricated panel used the staggered plies rather than the original pattern shown here.

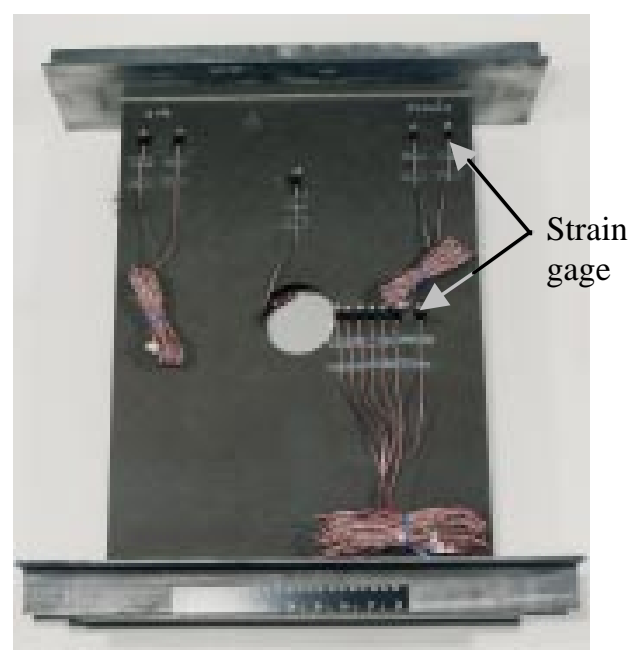

Fig. 12. Overlap specimen C3 prior to testing.

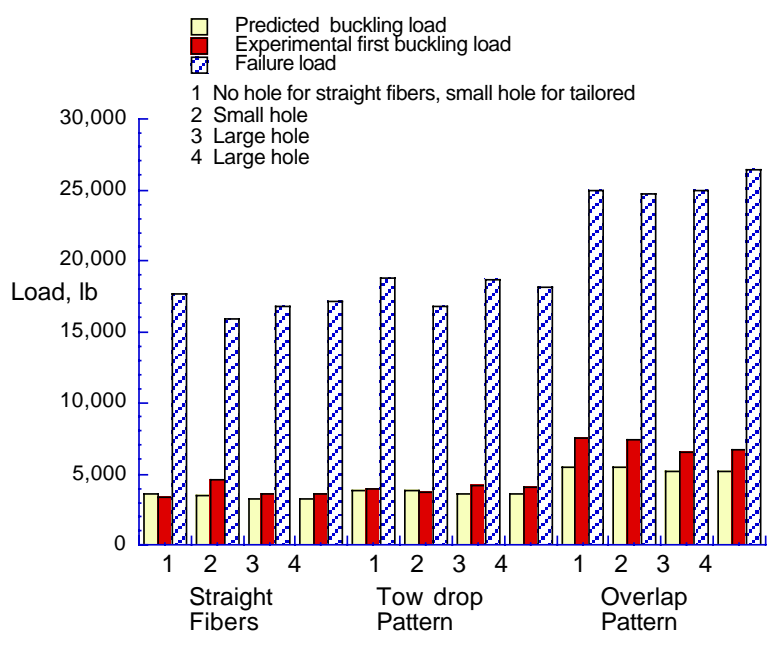

Fig. 13. Failure and buckling loads of tested specimens. 


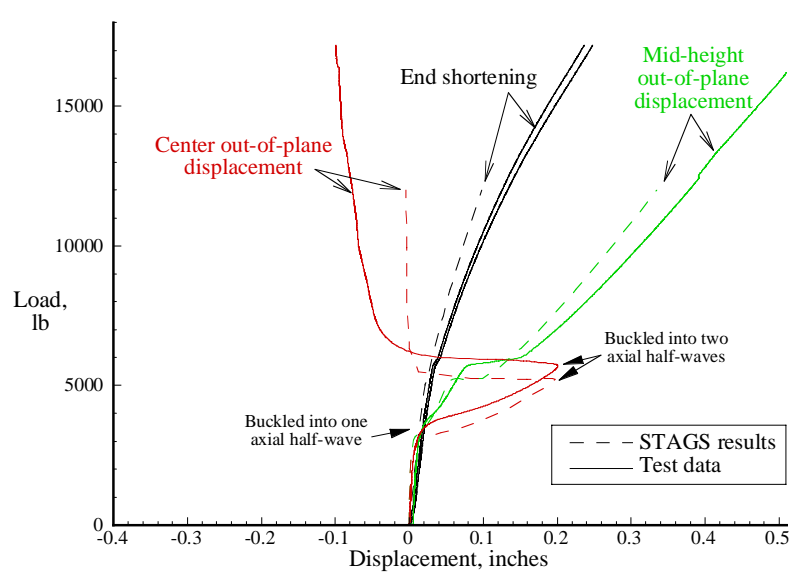

Fig. 14. Displacements for straight-fiber specimen A4.

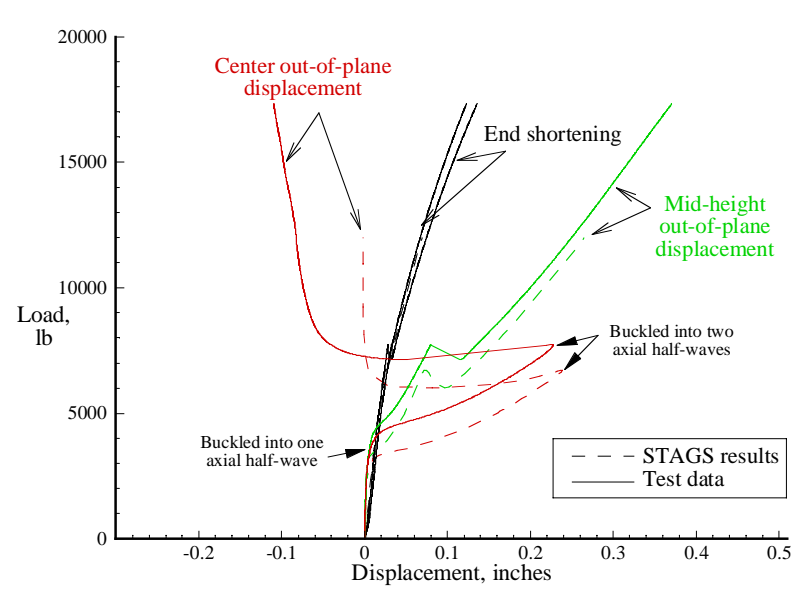

Fig. 15. Displacements for tow-drop specimen B3.

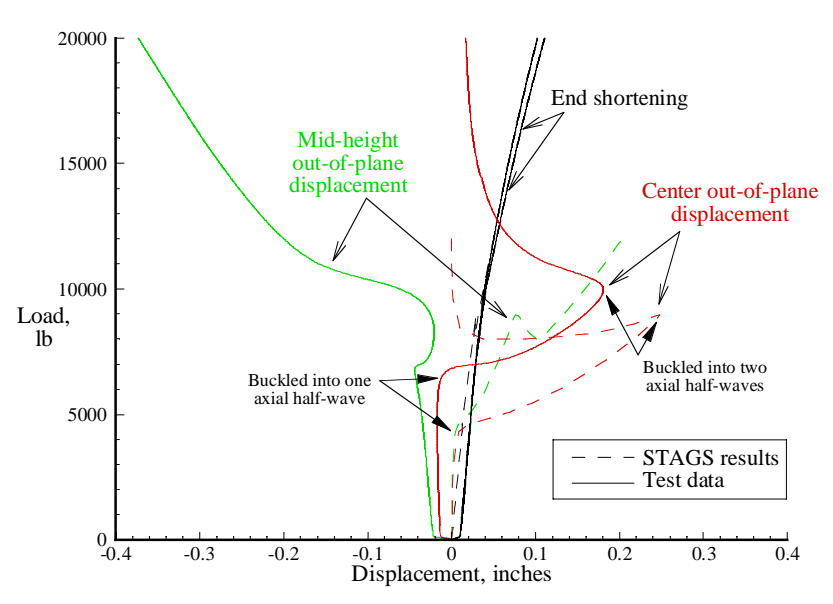

Fig. 16. Displacements for overlap specimen C3.

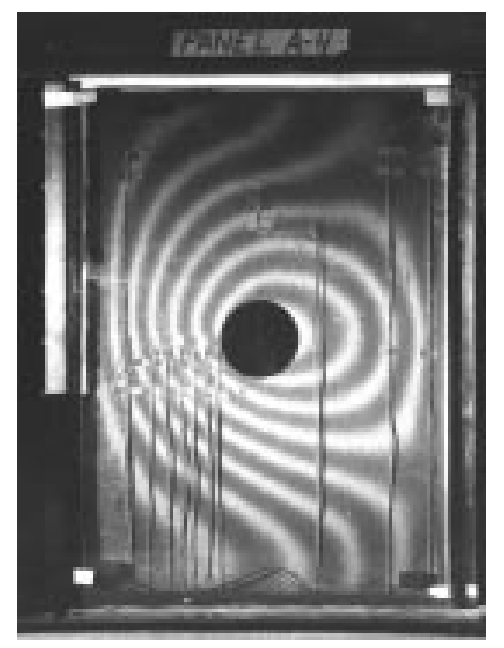

Fig. 17. Out-of-plane displacement straight-fiber specimen A4 subjected to a load of $4300 \mathrm{lb}$.

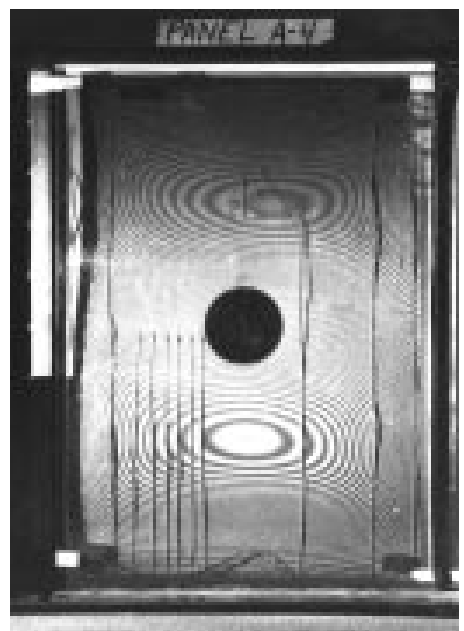

Fig. 18. Out-of-plane displacement pattern for straightfiber specimen A4 subjected to a load of 11,900 lb.

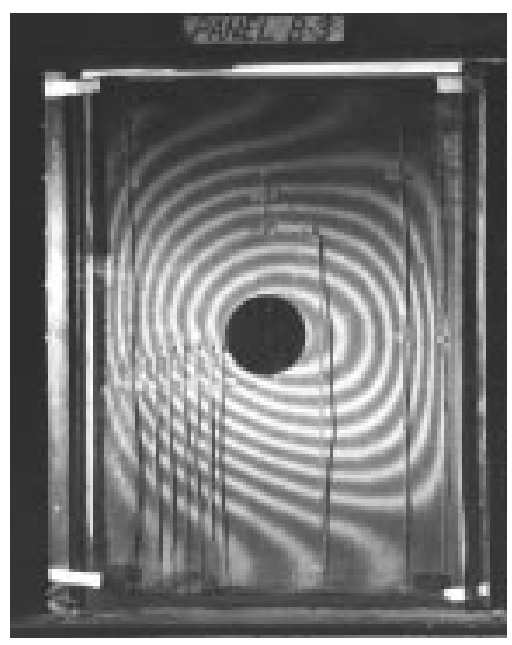

Fig. 19. Out-of-plane displacement pattern for tow-drop specimen B3 subjected to a load of $6200 \mathrm{lb}$. 


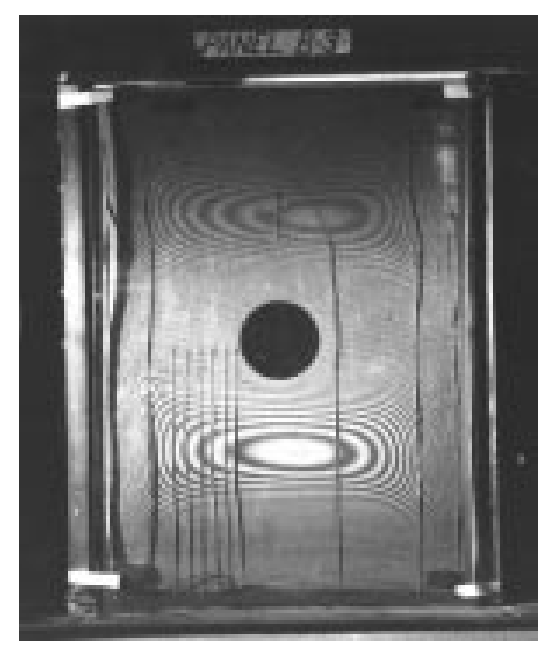

Fig. 20. Out-of-plane displacement pattern for tow-drop specimen B3 subjected to a load of 18,600 lb.

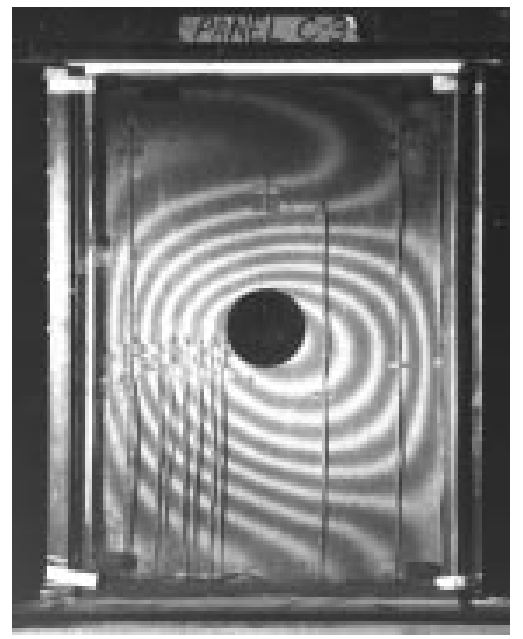

Fig. 21. Out-of-plane displacement pattern for overlap specimen C3 subjected to a load of $7700 \mathrm{lb}$.

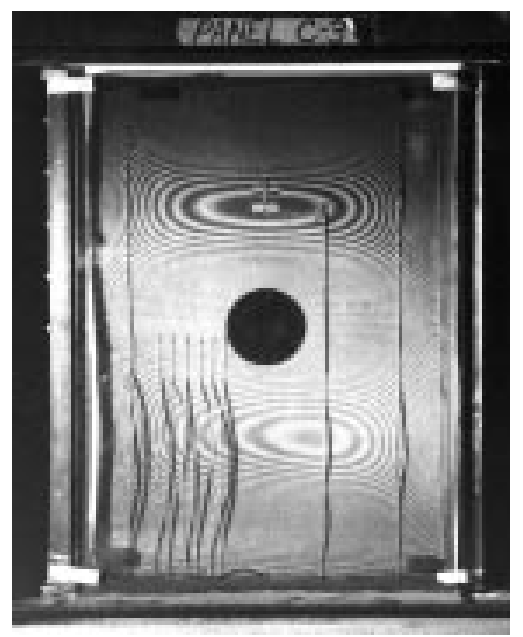

Fig. 22. Out-of-plane displacement pattern for overlap specimen C3 subjected to a load of 24,100 lb. 